\title{
Single-electron Transport Through Quantum Point Contact
}

\author{
G. Bilgeç Akyüzz, ${ }^{\mathrm{a}, *}$ A. Siddiki ${ }^{\mathrm{b}}$ \\ ${ }^{a}$ Adnan Menderes University, Faculty of Arts and Sciences, Physics Department, 09100, Aydin, Turkey \\ ${ }^{b}$ Mimar Sinan Fine Arts University, Faculty of Science and Letters, Physics Department, Bomonti-Sisli, 34380 Istanbul, Turkey
}

\begin{abstract}
Here, we employ a numerical approach to investigate the transport and conductance characteristics of a quantum point contact. A quantum point contact is a narrow constriction of a width comparable to the electron wavelength defined in a two-dimensional electron gas (2DEG) by means of split-gate or etching technique. Their properties have been widely investigated in the experiments. We define a quantum Hall based split-gate quantum point contact with standard gate geometry. Firstly, we obtain the spatial distribution of incompressible strips (current channels) by applying a self consistent Thomas-Fermi method to a realistic heterostructure under quantized Hall conditions. Later, time-dependent Schrodinger equation is solved for electrons injected in the current channels. The transport characteristics and time-evolutions are analyzed in the integer filling factor regime $(v=1)$ with the single electron density. The results confirm that the current direction in a realistic quantum point contact can be controllable with the external interventions.
\end{abstract}

\section{Introduction}

Developments in the quantum information processing technology have lead to intensive studies in the investigation of intrinsic properties of small-scale electronic devices. The conductance quantization is a fundamental phenomenon of electron transport in the low-dimensional structures and can be observed in a quantum point contact (QPC) which is constructed by geometric or electrostatic confinement of a two-dimensional electron gas. Therefore, a detailed understanding of the QPC conductance which represents cornerstone of mesoscopic physics and is of the prime importance via conductance through them is quantized at zero magnetic field [1]. So the properties of these small-scale devices have been generously investigated in the experiments such as Quantum Hall effect of the Mach Zender [4, 5], Aharanov-Bohm interferometers and the observation of 0.7 anomaly [2, 3]. Electrostatic and transport properties of the QPC's in quantized and non-quantized magnetic fields are also investigated early studies, comprehensively [1]. In a Quantum Hall device, the current carrying states are compose of with the Landau Level quantization and electron distribution in a Hall device depends on the pinning of the Fermi level to the highly degenerate Landau levels. According to self-consistent screening theory, 2DES contains two different kinds of regions namely incompressible

*Corresponding Author. Tel.: +902562128498; Fax: +902562135379

Email address: gonulbilgec@adu.edu.tr (G. Bilgeç Akyüz) 
(IS) and compressible strips (CS) [6, 7], which means if the Fermi level lies within a Landau level with the high density of state, system is called as compressible otherwise system is called as incompressible. In the compressible region, system has a flat potential profile and the screening property. In case of incompressible strips, system has constant electron density and generally a spatially varying potential because of the absence of the screening [1]. The current is transported by the scattering-free ISs when the quantum mechanical effects are smaller than the ISs widths [8]. Therefore, it is very important to deal with realistic constraining geometries due to boundary condition dictated properties of incompressible strips.

In the present study, we aimed to obtain a comprehensive understanding of the conductance of a standard QPC geometry in a quantum Hall device. We solved the 3D Poisson equation self-consistently and utilized ThomasFermi approximation to a heterostructure [9] taking into account the lithographically defined surface gates and we obtained the electron and potential distributions and incompressible strips ( $v=1$-current channels) under quantum Hall conditions. To describe the current channels, we use a model potential and a time-dependent solution to observe the conductivity of a wave packet injected into the channel. We have searched how is affected the QPC conductance with the interchannel distance, magnetic flux, channel depth and channel width. Additionally, we investigate and seek for the optimal conditions of a single-electron transport in a QPC geometry.

\section{Model and Methods}

The structural information is provided by Arslan et al [9] which consists of metallic surface gates (dark semielliptic regions) and a thin donor layer ( $\delta$-silicon doping) which provides electrons to the two-dimensional electron gas (2DES) (Fig 13). 2DES is confined to a thin area which is located at the interface of the heterostructure. The spatial distribution of the electron density is determined by the donors, the gate voltage $V_{g}$ and the gate shape. In a Quantum Hall device, the current carrying states result from the Landau level quantization, followed by level bonding at the edges. So the transport takes place through the edge states. The potential and charge distribution of the system under quantum hall conditions can be obtained by solving poisson equation self-consistently within Thomas-Fermi approximation. Electrostatic calculations are performed as in refs [9, 10] and the transport simulation has been carried by injecting single-electron wave packet to a static $2 \mathrm{D}$ potential.

In Figure 1 $\mathrm{b}$ b-d, we show the spatial distribution of the incompressible strips (ISs) at different magnetic fields and gate potentials. As explicitly seen, magnetic field (Fig_1/b-c) and gate potential $\left(V_{g}\right)$ determine the spatial distribution of the ISs (Fig1/d). The interchannel distance decrease while the ISs width increases with the increasing magnetic field. At lower magnetic fields, the strips are far apart and the conductance are weak or not present. So, it is expected that the wave packet remains in the incoming channel (lower left channel). To analyze the effects of internal distance, channel depth and channel width, we focus on realistic modeling of current channels along ISs following by a dynamical study on electron transport in the device. The channels are modeled by a 2D potential profile consisting of two curved pipes following the shape of the ISs in Figure $1 \mathrm{l} b-\mathrm{d})$. The distance between the left and right channels is 
varied as $d=0-1$ range (in atomic units).

The potential to transport corresponds to (ISs) with $v=1$ integer filling factor and these ISs carry the unbalanced electrical current. The potential minima of the channels are shown as solid lines in Figure 1 b- $\mathrm{d}$ and the cross section of these channels have a Gaussian form;

$$
V=-V_{0} \exp \left(-s^{2} / c^{2}\right)
$$

where $s$ is the perpendicular axis to the current-channel. $V_{0}=20$ (Hartree a.u. used throught) is the depth of the track which prevent the electrons from escaping the channels and $\mathrm{c}$ is the variable width parameter, initially set to 0.2 . It is useful to choose a gaussian form for the current channels because, it is a reasonable approximation for the magnetic enclosure at the bottom of the channel and this form enable to leak electron current coherent with the experimental case. Additionally, in the time-propagation, excess electron density on top of the Fermi background is used and is assumed to be static.

In the calculations, as an initial condition we set a single-electron wave packet in the lower-left corner of the incompressible strip and we track the density during the time propagation until we find back scattering from the corners of our finite simulation box. Thus, the direction of the current is the same in right and left channels (non-chiral transport) as in the non-equilibrium transport experiment. To provide a source-drain voltage to the current channel, it is applied a linear ramp potential $\left(V=-0.2 V_{0}\right)$ which accelerate the initial state. In the transport experiments, an external current is applied and a Hall potential is observed and this potential has the same slope in the two opposite corners. Therefore a potential drop occurs at the incompressible strips which are also observed in the some experiments $\llbracket 11$, 12].

The conductance as a function of the magnetic flux, the channel depth, interchannel distance and channel width is determined by calculating the probability $N_{r}$ to find the electron in the corners within the determined simulation time ( $t=14$ a.u) [14]. Time dependent density functional theory (TDDFT) is applied upon a 2D-real space grid for the time propagation of the electrons. The calculations have been performed by the OCTOPUS code package [13]. In the calculations, independent electron approximation has been used to completely neglect the interactions by using single electron wave-packet.

\section{Results}

Transport simulation is carried out by injecting single-electron wave packet into a static 2D potential. The potential minima of the channels (Fig,1/b-d) are modeled by a 2D potential profile following the shape of the IS. We consider that the interchannel distance (d) would be essentially controlled either with gates or magnetic field.

In our transport simulations, we track electron dynamics as a function of a magnetic flux which is applied the center of interchannel distance and added to the background magnetic field. In the first time-dependent simulations, we applied relative fluxes $\phi / \phi_{0}$ which is used in the range of $0.0-3.0$. In here, $\phi_{0}=h / e$ and $\phi=B \times A$, A define an area in which magnetic field is applied. The conductance of the system is considered as a function of the uniform 

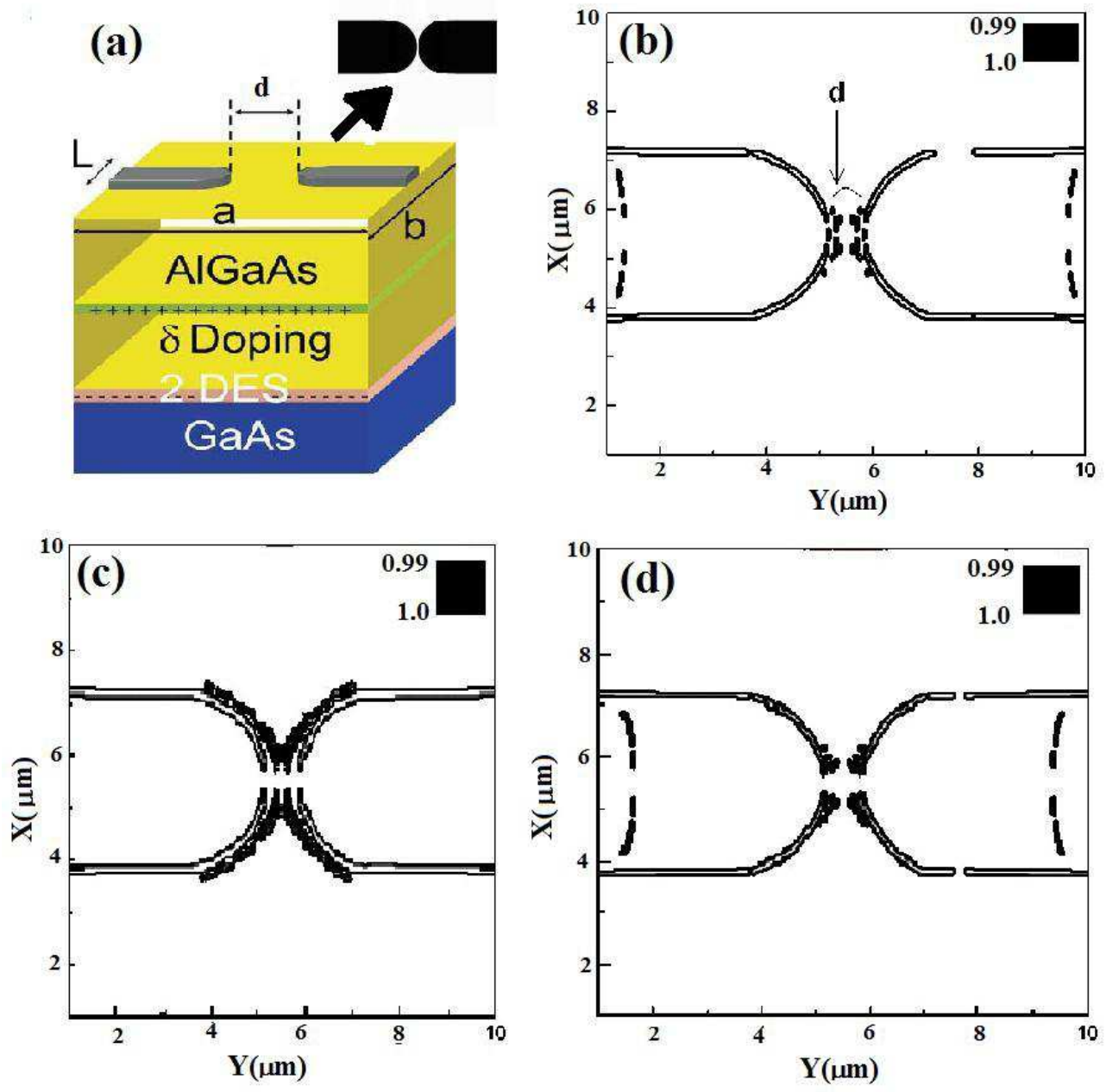

Figure 1: Spatial distribution of the incompressible strips calculated at (a)Heterostructure, the metallic gates are deposited on the surface. (b)Vgate $=1.7, \mathrm{~B}=2.5 \mathrm{~T}$, (c) Vgate $=1.7, \mathrm{~B}=5 \mathrm{~T}$ (d) Vgate=2.6, $\mathrm{B}=2.6 \mathrm{~T}$. It is expected that only one can observe the conductance at (d) conditions, whereas, the scattering between the edge states is presented in other cases.

and perpendicular magnetic field strength in the units of the enclosed flux quanta $\left(\phi / \phi_{0}\right)$. The wave packet with slope $V_{1}=-0.2 V_{0}$ is accelerated along the current channels. To analyze the transport characteristics in detail, we calculate the conductance as a function of the magnetic flux for different interchannel distances $(d=0.3-1.0)$ in Figure 2

The panels in Figure 2 show conductance for different interchannel distances at the corners. In this analysis, we compare the electron density at the different corners (top-right, down and top-left) and obtain distinctive characteristics for different conditions. Maximum probability has been observed in the lower-right channel conductance for the minimum interchannel distance $(\mathrm{d}=0.3)$ (Fig.2a). It is observed a small peak at nearly $\phi / \phi_{0}=2.6$ value for the 

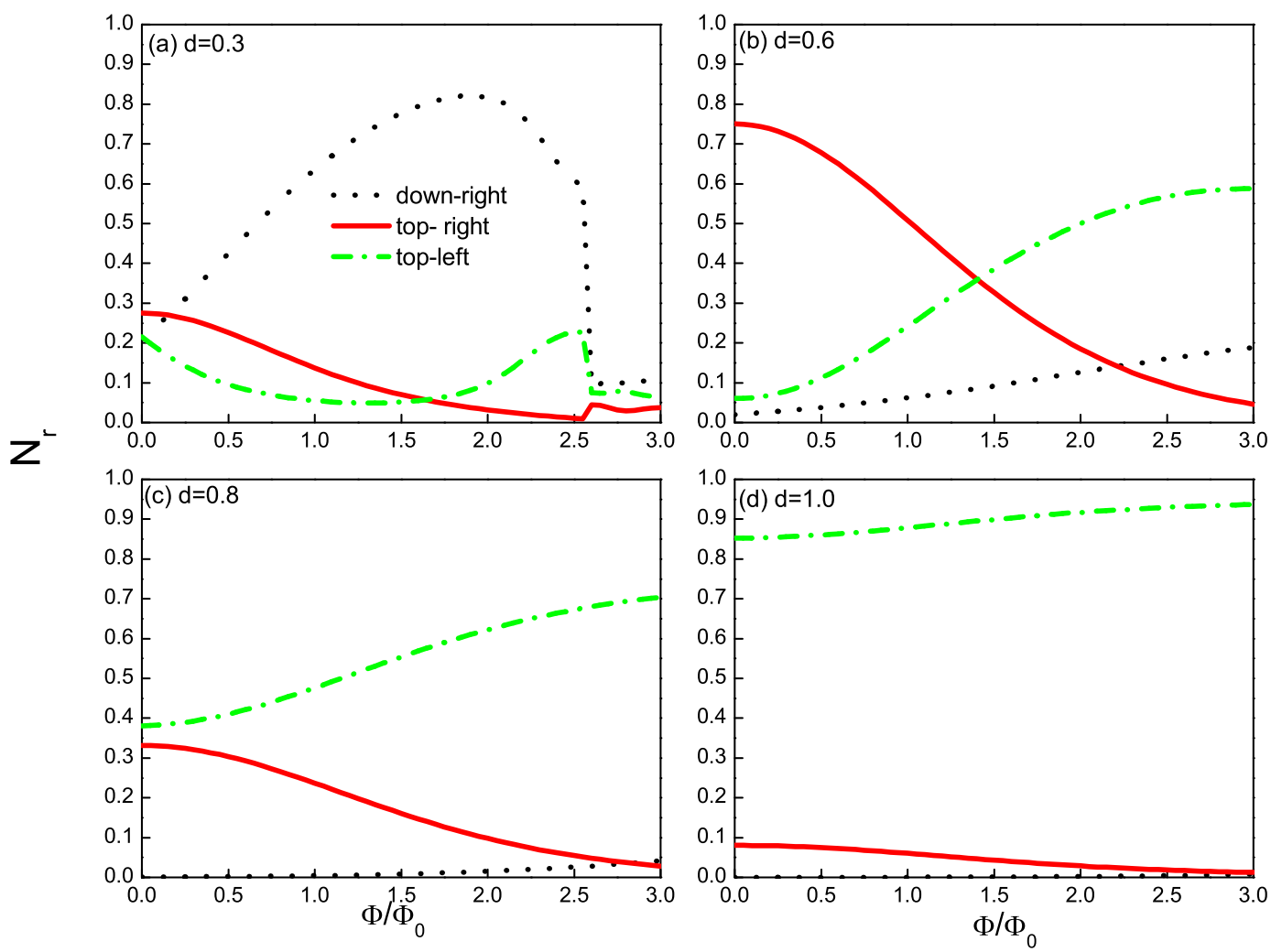

Figure 2: Estimated conductance $\left(N_{r}\right)$ as a function of the magnetic flux in the center of QPC-gates for different interchannel distances.

top-right conductance and at this critical magnetic flux value, conductance probabilities of other current channels show a sharp decline. Magnetic flux applied the center of the interchannel distance partly prevent to transport to the right channel and go ahead to top-left above $\phi / \phi_{0}=2.6$ value. Maximum conductance is obtained at nearly $\phi / \phi_{0}=2$ value, for down-right channel. A similar drop is also observed for the top-left channel conductance for this interchannel distance. This value can be called as critical current value because it is observed a considerable decline in the probabilities of the channels above the critical value. After this critical current value, largely amount of singleelectron wave packet remains in the lower-left channel (incoming channel), but nevertheless probability do not vanish in the three region. Conductance characteristic of the QPC with the $d=0.6$ interchannel distance are more intelligible than $d=0.3$ (Fig.2(a)-(b)). In this situation, top-right conductance is decreased while the left-top one is increased with the increasing magnetic flux which is applied the center of the interchannel distance. This magnetic flux prevent the transport of the electron wave-packet to the top-right channel. Although, the transport schema of the $d=0.8$ has 
similar characteristics with the transport of the $d=0.6$, a discrepancy in the conductance graph is also observed for $d=0.8$ interchannel distance. For $d=0.8$, conductance probabilities are lower than the $d=0.6$ interchannel distance. The changing rates of the right and left top channel conductance probabilities are almost seen equal. It is also seen that the probability $\left(N_{r}\right)$ of the finding the electron in the right-down corner is very weak.

For $d=1.0$ interchannel distance (Fig $2 \mathrm{~d}$ ), it is not observed a important effect of the magnetic flux on the transport to the right channels. Single-electron wave packet remain the left channel and probability which has already small value decrease with the increasing magnetic flux. So, in these conditions, interchannel transport is completely blocked near the $\phi / \phi_{0} \approx 3.0$ value. This behavior is explained by the reason of wide interchannel distance $(d=1.0)$ for the electron wave packet to the right channels.

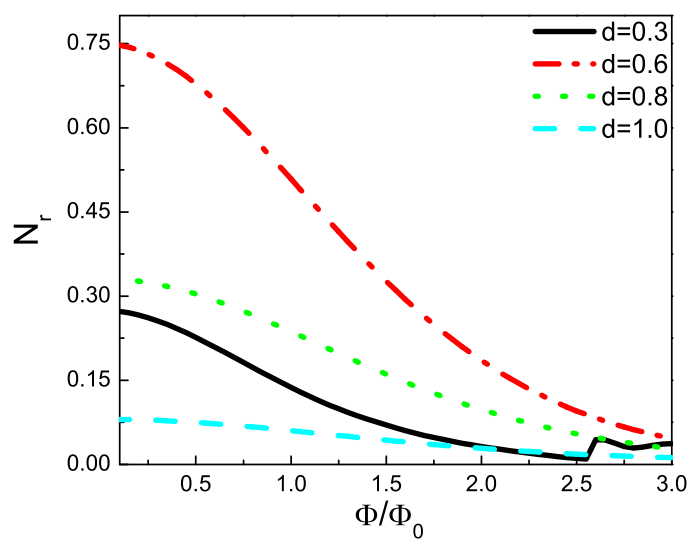

Figure 3: Estimated conductance (top-right channel) $\left(N_{r}\right)$-flux graph at different distances

To determine the optimal interchannel distance for conductance which correspond here to the electron density transferred to the top-right channel, we plot the conductance probability $\left(N_{r}\right)$ as a function of the magnetic flux for different interchannel distances (Figure 3). Optimal distance is determined as $\mathrm{d} \sim 0.6$ interchannel distance from this analysis. At smaller and larger values of interval distances, conductivity probability is significantly decreased. This result confirm similar transport studies [10,15]. In general, the top-right conductance probability of the QPC devices decrease linearly with the increasing magnetic flux applied to the center of QPC. However, it is observed a peak for the $d=0.3$ interchannel distance above the nearly $\phi / \phi_{0}=2.6$ magnetic flux value. The reason of the observation such a peak, it may be sharp decline in the conductivity for other current channels for this interchannel distance.

Conductance-channel width analysis show another property of the QPC (Fig 4). It is seen that the optimal distance also depends on the channel width. The conductivities of the devices with the interchannel distances $d=0.6$ and $d=0.3$ show peaks while the conductivities of the other devices show dips at nearly 0.2-0.3 channel width range. At the same time, probabilities vary smoothly after the nearly $c=0.5$ value for $d=0.6$ and $d=0.8$.

Another remarkable observation of $N_{r}-c$ graph is that maximum conductances are observed for $\mathrm{d}=0.3-0.6$ 
interchannel distances while minimum conductances are observed for $\mathrm{d}=0.8-1.0$ interchannel distances at small channel width $(\sim 0.2)$. Therefore, the optimal top-right conductance probability is determined at nearly 0.2 channel width value for $d=0.6$ interchannel distance and at larger channel widths $(c>0.5)$ for $d=0.8$ interchannel distance. Above the nearly $c=0.2$ value, conductance increase with the increasing channel width in this $c$ range for $d=1$.0distance. Additionally, it is seen that the conductance probabilities of $d=0.6$ and $d=1.0$ interchannel distance coincide for wide channel widths $(c>1.3)$. The reason of this coinciding is that the widening current channels get closer to each other. Probability oscillate with small amplitude with the increasing channel width for $d=0.3$ interchannel width. Therefore, we emphasize that the channel width is as important as interchannel distances on the QPC conductivities.

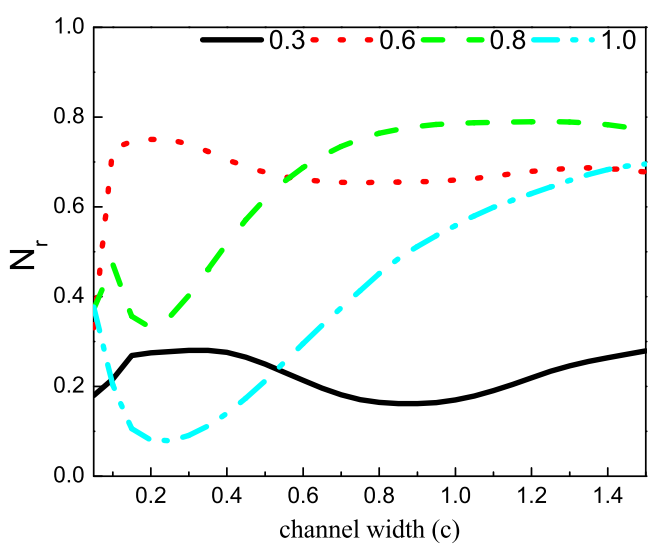

Figure 4: Estimated conductance (top-right channel- $\left.N_{r}\right)$ at zero magnetic field $\left(\phi / \phi_{0}=0\right)$ as a function of channel width $(c)$ at different interchannel distances (d), $V=0.2 V_{0}$. The optimal distance for the conductivity probability also depends on channel width.

Finally, Figure 5 shows the top-right conductance of the QPC for different channel depth values $V\left(V_{0}\right)$ at zero magnetic flux value. The conductance probabilities increase with increased potential depth for the devices with $d=0.3$ and $d=0.6$ interchannel distances. Additionally, the results in Figure 5 confirm that the optimal interval distance is 0.6 and maximum probability is obtained in $V=0.18 V_{0}$ value for optimal interchannel distance. For $d=0.3$ interchannel distance, changes in the conductance are non-crucial, $N_{r}$ oscillates with small amplitudes. Conductance probability to the right channel decrease with the increasing channel depth for $d=0.8, d=1.0$, because it is think that the single electron wave packet are confined in the current channel. However, the conductance probabilities almost linearly decrease with the increased channel depth for the devices with $d=0.8$ and $d=1.0$ interchannel distances. So, the increased channel depth has a negative effect on the conductance for large interchannel distances. This confining of electron wave packet is also affects the optimal interchannel distance $(d=0.6)$ for large channel depths and it is observed an decreasing in the conductance probability. So, it can be concluded that if the interchannel distances will 
be broad, channel depth must be low for the high conductance probability.

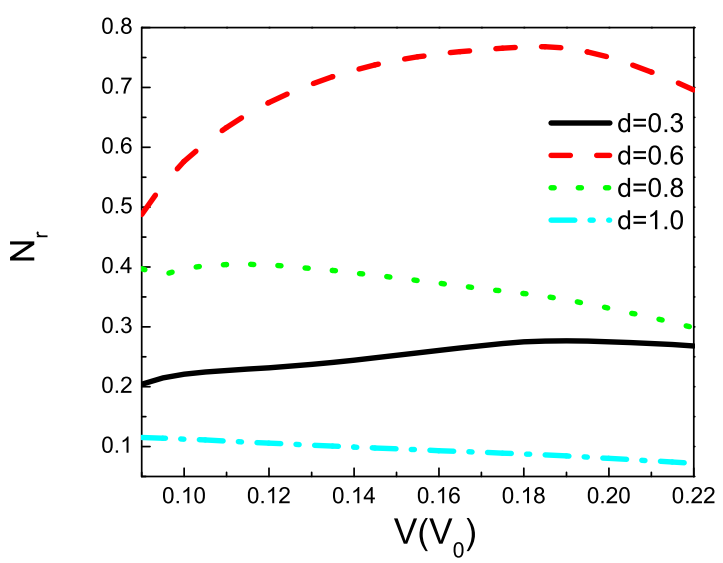

Figure 5: Estimated conductance (top-right channel- $\left.N_{r}\right)$ at zero magnetic flux $\left(\phi / \phi_{0}=0\right)$ as a function of channel depth values for $\mathrm{c}=0.2$ values.

\section{Conclusion}

We have performed a single-electron wave packet propagation scheme by using time dependent density functional theory to study electron transport in the integer filling factor $(v=1)$ regime for a realistic Quantum point contact geometry. We observe distinctive QPC conductivity behaviors, where their amplitudes strongly depend on the interchannel distances, channel widths and channel depth values. For the top-right channel conductivity, the interchannel distance has an optimal value $d \sim 0.6$ and $d \sim 0.3$ for the down-right channel conductivity when the conductance is maximized. However, we obtain an optimal transport condition depending on channel width for the top-right channel conductivity. Eventually, we obtain distinct conductivity graphs in all cases and all directions. So, we conclude that the direction and amplitude of a single electron wave packet is completely controllable by the external factors as magnetic flux, channel depth and width etc. We are sure that the numerical results presented here will pave path for further experimental efforts in the QPC conductivities.

\section{References}

[1] A.Siddiki, F. Marquardt, Phys. Rev. B, 75,045325 (2007)

[2] K. J. Thomas, J. T. Nicholls, M. Y. Simmons, M. Pepper, D. R. Mace, and D. A. Ritchie, Phys. Rev. Lett. 77, 135 (1996).

[3] K. Thomas, J. Nicholls, M. Simmons, M. Pepper, D. Mace, and D. Ritchie, Phys. Rev. Lett. 77, 135 (1996).

[4] Y. Ji, Y. Chung, D. Sprinzak, M. Heiblum, D. Mahalu, and H. Shtrikman, Nature 422, 415 (2003).

[5] I. Neder, M. Heiblum, Y. Levinson, D. Mahalu, and V. Umansky, Phys. Rev. Lett. 96, 016804 (2006).

[6] D.B. Chklovskii, B.I.Shklovskii, and L.I. Glazman, Phys. Rev. B 46, 4026 (1992) 
[7] A. Siddiki and R. R. Gerhardts, Phys. Rev. B 68, 125315 (2003)

[8] A.Siddiki, R.R. Gerhardts, Phys. Rev. B 70 (2004) 195335.

[9] S. Arslan, E. Cicek, D. Eksi, S.Aktas, A. Weichselbaum and A. Siddiki, Phys.Rev.B 78, 125423 (2008).

[10] V.Kotimaki, E.Cicek,A.Siddiki and E.Rasanen, New.Phys.14,053024 (2012).

[11] E. Ahlswede, P.Weitz, J.Weis, K.von Klitzing, and K. Eberl, Physica B 298, 562 (2001).

[12] E. Ahlswede, J.Weis, K.von Klitzing, and K. Eberl, Physica E 12, 165 (2002.

[13] M.A.L. Marques, A. Castro, G.F. Bertsch, A.Rubio, Comput.Phys.Commun.151, 60 (2003); A.Castro, H. Appel, M. Oliveira, C. A. Rozzi, X. Andrade,F. Lorenzen, M.A.L. Marques, E.K.U. Gross, and A. Rubio, Phys. Stat. Sol. (b) 243, 2465 (2006).

[14] V.Kotimaki and E.Rasanen,Phys.Rev.B 81245316.

[15] A. Salman, V.Kotimaki, A.Siddiki, E.Rasanen, EPJ B, 86, 155 (2013). 